\title{
HIDDEN MODALITIES IN ALGEBRAS WITH NEGATION AND IMPLICATION
}

\author{
JOUNI JÄRVINEN, MICHIRO KONDO, JORMA K. MATTILA \\ AND SÁNDOR RADELECZKI
}

\begin{abstract}
. Łukasiewicz 3-valued logic may be seen as a logic with hidden truthfunctional modalities defined by $\diamond A:=\neg A \rightarrow A$ and $\square A:=\neg(A \rightarrow \neg A)$. It is known that axioms (K), (T), (B), (D), (S4), (S5) are provable for these modalities, and rule $(\mathrm{RN})$ is admissible. We show that, if analogously defined modalities are adopted in Łukasiewicz 4-valued logic, then (K), (T), (D), (B) are provable, and $(\mathrm{RN})$ is admissible. In addition, we show that in the canonical $n$-valued ŁukasiewiczMoisil algebras $L_{n}$, identities corresponding to (K), (T), and (D) hold for all $n \geq$ 3 and $\square 1=1$. We define analogous operations in residuated lattices and show that residuated lattices determine modal systems in which axioms (K) and (D) are provable and $\square 1=1$ holds. Involutive residuated lattices satisfy also the identity corresponding to $(\mathrm{T})$. We also show that involutive residuated lattices do not satisfy identities corresponding to (S4) nor (S5). Finally, we show that in Heyting algebras, and thus in intuitionistic logic, $\diamond$ and $\square$ are equal, and they correspond to the double negation $\neg \neg$.
\end{abstract}

\section{Some historical REMARKS}

We refer here to Nicholas Rescher, who has considered the history of many-valued logics in Chapter 1 of his book Many-valued Logic [17], and William and Martha Kneale's book The Development of Logic [11]. Rescher ([17], pp. 1-2) writes:

"In Chap. 9 of his treatise De interpretatione Aristotle discussed the truth status of alternatives regarding "future-contingent" matters, whose occurrence - like that of the sea battle tomorrow - is not yet determinable by us, and may indeed actually be undetermined. His views on the matter are still disputed, but many commentators, both in antiquity and later, held him to maintain that propositions about future contingents, like that asserting the occurrence of the sea battle tomorrow, are neither actually true nor actually false, but potentially either, thus having - at least

$M S C$ (2010): primary 03B50; secondary 03B45, 03B52, $68 \mathrm{~T} 37$.

Keywords: Łukasiewicz 3-valued logic, Łukasiewicz-Moisil algebras, fuzzy sets, rough sets, residuated lattices, Heyting algebras, intuitionistic logic.

The research of the fourth author was carried out as part of the TAMOP-4.2.1.B10/2/KONV-2010-0001 project supported by the European Union, co-financed by the European Social Fund. 
prior to the event - a third, indeterminate truth status. The acceptance of the principle of bivalence was, in antiquity, closely bound up with the doctrine of determinism."

The indeterminate truth status has been the main question. How to model it? A solution to this question gives the third truth value. Several attempts have appeared. These attempts have centralized into two different groups of modalities, namely alethic and probabilistic modalities. The alethic modalities are (see Rescher $[17$, p. 3])

- necessarily true,

- contingently (i.e., actually but not necessarily) true,

- contingently false,

- necessarily false.

The probabilistic modalities are

- certainly true,

- probably true,

- probably false,

- certainly false.

These modalities were studied already in the ancient time of the development of logic, that is, in the period from Plato's Academy to the second half of 19th century. Particularly, Aristoteles constructed a theory of modal statements and modal syllogisms (see Subsection 7 Aristotle's Modal logic of Section II in Kneale \& Kneale [11]).

According to Rescher, the early historical period is the period 1875-1916. The systematic study of many-valued logic began in this period. According to Rescher, the founding fathers of many-valued logic were Scotsman Hugh MacColl (1837-1909), American Charles Sanders Peirce (1839-1914), and Russian Nikolai A. Vasilév (1880-1940).

MacColl sketched a system of propositional logic in which propositions can take on several distinct truth values, like the modal values of certainty, impossibility, and variability (contingency). He characterized his system as a "logic of three dimensions". So, MacColl counted mainly on probabilistic modalities.

Peirce approached the ideas of many-valued logic from several points of departure. For one thing, he conceived of the idea of a neutral truth value in the traditional context of Aristotle's problem of future contingency. Peirce seemed to study mainly alethic modalities, and so did Vasilév, for creating indeterminate truth values.

In the period 1910-1914 Vasilév published several papers about what he called "imaginary non-Aristotelian logics". He described his work as an attempt to do for Aristotelian logic the same as an earlier professor at Kazan University, Nikolai Lobachevsky, had done for Euclidean geometry. Vasilév wanted to see what logical principles could be changed or eliminated from logic without its ceasing to be logic.

According to Rescher, the period 1920-1932 is called the pioneering era in the development of many-valued logics. Rescher writes: 
"The actual inauguration of many-valued logic must be dated from the pioneering papers of the Pole Jan Eukasiewicz and the American Emil L. Post, published in the early 1920s, in which the first development systematizations of many-valued logic are presented."

Some history of these pioneering things is also given in Subsection 5 of Section IX of Kneale \& Kneale [11].

Łukasiewicz first published his 3-valued system of logic in a lecture before the Polish Philosophical Society in Lwów in 1920. Łukasiewicz' motivation for defining the derived connectives disjunction and conjunction by means of the primitive connectives negation and implication, and further the modal operation possible, on the suggestion of his pupil A. Tarski, is described largely in Subsection 5 of Section IX of Kneale \& Kneale [11].

What is followed from those earlier periods can be seen in the next period 1932-1965. The theory and applications of many-valued logic were developed along a great variety of lines during the period 1920-1932. Various writers have recently carried further the study of the initial 3-valued logic of Łukasiewicz and his later many-valued generalizations of it. In this regard, apart from the continuing contributions of Łukasiewicz himself and the results of Wajsberg, the work of other logicians of the Polish school - Alfred Tarski, Jerzy Słupeci, Bolesław Sobociński, and others - is especially important. The same can be said about the joint works of James Barkley Rosser and Atwell R. Turquette, and papers by Emil L. Post, Helena Rasiowa, Allan Rose, and Chen Chung Chang.

In the late 1950s, the interest in many valued logics appeared to decrease. The main reason may have been that further applications did not appear, especially in the field of philosophy.

A new outcome of many-valued logics appeared after Lotfi A. Zadeh's introduction to fuzzy set theory. Particularly, algebraic theories of many-valuation began to develop again. A Polish counterpart to fuzzy sets, namely rough sets was due to Zdzisław Pawlak. A new topic of research, soft computing was formed by the connection of fuzzy sets, rough sets, probability, neural networks and genetic algorithms into the same field. So, a new period began, which we may call recent period or soft computing period (1965-).

\section{2. $\mathrm{Ł}_{3}$ AS A MODAL LOGIC}

Three-valued system of propositional calculus was constructed by Jan Łukasiewicz. In his 3 -valued logic, propositions can have the truth values $T, I$, and $F$, where $T$ stands for "true", $I$ means "neither true or false", called also "intermediate", and $F$ denotes "false". These values may be ordered by $F<I<T$. Obeying the philosophical tradition, we mostly use these letter symbols for the truth values instead of numeric symbols, such as $0, \frac{1}{2}$, and 1 .

Łukasiewicz chose the connectives of negation and implication as primitives and defined them in his 3-valued logic, denoted by $\mathrm{E}_{3}$, as in Table 1.

In 1930's, Mordchaj Wajsberg proposed an axiomatization for $\mathrm{E}_{3}$ :

$(\mathrm{Ax} 1) \quad A \rightarrow(B \rightarrow A)$, 


\begin{tabular}{|c|c|}
\hline & $\neg$ \\
\hline$T$ & $F$ \\
$I$ & $I$ \\
$F$ & $T$ \\
\hline
\end{tabular}

\begin{tabular}{|c|ccc|}
\hline$\rightarrow$ & $T$ & $I$ & $F$ \\
\hline$T$ & $T$ & $I$ & $F$ \\
$I$ & $T$ & $T$ & $I$ \\
$F$ & $T$ & $T$ & $T$ \\
\hline
\end{tabular}

Table 1. Truth tables for negation (left) and implication (right).

$(\mathrm{Ax} 2)(A \rightarrow B) \rightarrow((B \rightarrow C) \rightarrow(A \rightarrow C))$,

$(\mathrm{Ax} 3)((A \rightarrow B) \rightarrow B) \rightarrow((B \rightarrow A) \rightarrow A)$,

$(\mathrm{Ax} 4)(\neg B \rightarrow \neg A) \rightarrow(A \rightarrow B)$.

The only rule of inference is modus ponens:

(MP) $\frac{A \quad A \rightarrow B}{B}$.

A proof is any finite sequence of formulas, each of which is an axiom or an immediate consequence, by applying (MP), of preceding formulas of the sequence. Any proof is said to prove its last formula, which is called a theorem or provable formula.

A valuation is a mapping $v$ assigning to each propositional variable $p$ its truth value $v(p) \in\{F, I, T\}$. Every valuation $v$ extends canonically to all formulas by

$$
v(\neg A)=\neg v(A) \quad \text { and } \quad v(A \rightarrow B)=v(A) \rightarrow v(B)
$$

A formula $A$ is valid, if $v(A)=T$ for all valuations $v$. As proved by Wajsberg, the axiom system $(\mathrm{Ax} 1)-(\mathrm{Ax} 4)$ with the rule modus ponens is complete with respect to the 3-element chain equipped with the operations presented in Table 1, that is, a formula of this system is provable if and only if it is valid.

Łukasiewicz also introduced 3-valued modal operations of possibility and necessity (see [17, p. 25] for further details) presented in Table 2.

\begin{tabular}{|c|c|c|}
\hline & $\diamond$ & $\square$ \\
\hline$T$ & $T$ & $T$ \\
$I$ & $T$ & $F$ \\
$F$ & $F$ & $F$ \\
\hline
\end{tabular}

Table 2. Truth tables for $\diamond$ and $\square$.

Based on the truth-table semantics of Table 2, we can define valuations for the formulas $\diamond A$ and $\square A$ by setting

$$
v(\diamond A)=\diamond v(A) \quad \text { and } \quad v(\square A)=\square v(A),
$$

for any formula $A$.

Notice that $\square$ and $\diamond$ are mutually dual, that is, $\square A \equiv \neg \diamond \neg A$ and $\diamond A \equiv \neg \square \neg A$ for all formulas $A$, where $A \equiv B$ means that the formulas $A$ and $B$ are semantically equivalent, that is, $v(A)=v(B)$ for all valuations $v$. A complete analysis of 3valued valuations and evaluation rules in $\mathrm{E}_{3}$ is given, e.g., in Mattila [13]. 
Alfred Tarski, being at that time Lukasiewicz' assistant, found out that the formula

$$
\neg A \rightarrow A
$$

has exactly the same truth table as the formula $\diamond A$. Analogously, it is easy to see that the formulas $\neg(A \rightarrow \neg A)$ and $\square A$ have identical truth tables. The above then means that in $\mathrm{E}_{3}$, the modalities $\diamond$ and $\square$ can be defined in terms of the negation $\neg$ and the implication $\rightarrow$ by setting

$$
\diamond A:=\neg A \rightarrow A \quad \text { and } \quad \square A:=\neg(A \rightarrow \neg A) .
$$

Thus, $\mathrm{E}_{3}$ can be viewed as a logic of hidden modalities.

In the sequel, we use (1.2) as the definitions for truth-functional modal operators. Recall that a connective is truth-functional if and only if the truth value of any compound statement obtained by applying that connective is a function completely determined by the individual truth values of the constituent statements that form the compound.

Most of the so-called normal modal logical systems can be characterized in terms of the following axiom schemata:

(K) $\square(A \rightarrow B) \rightarrow(\square A \rightarrow \square B)$,

(T) $\square A \rightarrow A$,

(B) $A \rightarrow \square \diamond A$,

(D) $\square A \rightarrow \diamond A$,

(S4) $\square A \rightarrow \square \square A$,

(S5) $\diamond A \rightarrow \square \diamond A$.

In addition, the inference rule (RN) is usually included:

(RN) $\frac{A}{\square A}$.

First, we consider these schemata in the framework of the logic $\mathrm{E}_{3}$. The next proposition can be easily verified by applying truth-tables (cf. [13]).

Proposition 2.1. Eukasiewicz 3-valued logic $\mathrm{E}_{3}$ is sound with respect to axioms (K), (T), (B), (D), (S4), (S5), that is, they are valid formulas. Additionally, the rules $(\mathrm{MP})$ and $(\mathrm{RN})$ preserve validity.

It is well known that the propositional two-valued modal logic is not truthfunctional. Łukasiewicz' motivation for his 3-valued logic was to construct a truthfunctional logic for modal operators. In that task he succeeded completely.

We note that if these definitions are done in propositional two-valued logic, then $\diamond A \equiv A \equiv \square A$ for all formulas $A$. In Section 4 , we study the properties of modalities (1.2) in Eukasiewicz-Moisil algebras, and Section 4 is devoted to the operators in residuated lattices and Heyting algebras generalizing the above 3 -valued system.

\section{LuKASIEWICZ-Moisil ALGEBRAS}

In 1940, Grigore Moisil introduced the $n$-valued Lukasiewicz algebras. Nowadays, these algebras are commonly called as Łukasiewicz-Moisil algebras. They are distributive lattices with a De Morgan negation and $n-1$ modal operators fulfilling the axioms given in Definition 3.2 defined on them. 
First, we recall the definition of De Morgan algebras from [1].

Definition 3.1. A De Morgan algebra is an algebra $(L, \vee, \wedge, \neg, 0,1)$, where $(L, \vee, \wedge, 0,1)$ is a bounded distributive lattice and $\neg$ is a unary operator satisfying the conditions:

(DM1) $\neg x \vee \neg y=\neg(x \wedge y)$,

(DM2) $\neg x \wedge \neg y=\neg(x \vee y)$,

(DM3) $\neg \neg x=x$.

Note that De Morgan algebras are also called quasi-Boolean algebras (see, e.g., $[16])$.

The following definition of $n$-valued Lukasiewicz-Moisil algebras can be found in [5], for example.

Definition 3.2. An $n$-valued Lukasiewicz-Moisil algebra, for $n \geq 2$, is a de Morgan algebra $(L, \vee, \wedge, \neg, 0,1)$ with unary operators $\phi_{1}, \phi_{2}, \ldots, \phi_{n-1}$ fulfilling the conditions:

(LM1) $\phi_{i}(x \vee y)=\phi_{i}(x) \vee \phi_{i}(y)$ for all $1 \leq i \leq n-1$.

(LM2) $\phi_{i}(x) \vee \neg \phi_{i}(x)=1$ for all $1 \leq i \leq n-1$.

(LM3) $\phi_{i}\left(\phi_{j}(x)\right)=\phi_{j}(x)$ all $1 \leq i, j \leq n-1$.

(LM4) $\phi_{i}(\neg x)=\neg \phi_{n-i}(x)$ for all $1 \leq i \leq n-1$.

(LM5) $\phi_{1}(x) \geq \phi_{2}(x) \geq \cdots \geq \phi_{n-1}(x)$.

(LM6) If $\phi_{i}(x)=\phi_{i}(y)$ for all $1 \leq i \leq n-1$, then $x=y$.

The condition (LM6) is called the determination principle. Let us mention that there exists also a variant of the definition in which condition (LM5) is replaced by the condition

$$
\phi_{1}(x) \leq \phi_{2}(x) \leq \cdots \leq \phi_{n-1}(x) .
$$

Note also that an equational characterization of $n$-valued Łukasiewicz-Moisil algebras can be found in [1].

In particular, for 3-valued Łukasiewicz-Moisil algebras, we have that

$$
\phi_{1}(x)=\neg \phi_{2}(\neg x)
$$

by (Ł4), that is, $\phi_{1}$ and $\phi_{2}$ are dual. This means that the conditions of Definition 3.2 can be presented in a simpler form by using only one operator $\diamond=\phi_{1}$ corresponding to "possibility".

Example 3.3. Three-valued Łukasiewicz-Moisil algebras have an interesting connection to rough sets introduced by Zdzisław Pawlak in [15]. In rough set theory, knowledge about objects is given in terms of an equivalence relation $\approx$, which is interpreted so that two objects $x$ and $y$ of some universe of discourse $U$ are $\approx$-related, that is, $x \approx y$ if and only if $x$ and $y$ are indiscernible with respect to their properties known by us. Let $[x]_{\approx}$ denote the $\approx$-class of $x \in U$, that is, the elements of $U$ that have exactly the same properties as $x$. The lower approximation of $X \subseteq U$ is defined by

$$
\underline{X}=\left\{x \in U \mid[x]_{\approx} \subseteq X\right\},
$$

and the upper approximation of $X$ is defined as

$$
\bar{X}=\left\{x \in U \mid[x]_{\approx} \cap X \neq \emptyset\right\} .
$$


The set $\underline{X}$ can be interpreted as a set of elements that certainly are in $X$ in view of the knowledge restricted by $\approx$, because if $x \in \underline{X}$, then all elements that are indiscernible with $x$ are also in $X$. Analogously, $\bar{X}$ may be viewed as the set of elements possibly in $X$ in view of the knowledge $\approx$, because if $x \in \bar{X}$, there exists at least one element $y \in X$ such that $x \approx y$. The set of all rough sets is then defined as

$$
\mathcal{R S}=\{(\underline{X}, \bar{X}) \mid X \subseteq U\} .
$$

As noted in [14], on $\mathcal{R S}$, a three-valued Łukasiewicz-Moisil algebra

$$
(\mathcal{R S}, \vee, \wedge, \diamond, \square, \neg, 0,1)
$$

can be defined by setting for all $X, Y \subseteq U$ :

$$
\begin{aligned}
(\underline{X}, \bar{X}) \vee(\underline{Y}, \bar{Y}) & =(\underline{X} \cup \underline{Y}, \bar{X} \cup \bar{X}) ; \\
(\underline{X}, \bar{X}) \wedge(\underline{Y}, \bar{Y}) & =(\underline{X} \cap \underline{Y}, \bar{X} \cap \bar{X}) ; \\
\triangleright(\underline{X}, \bar{X}) & =(\bar{X}, \bar{X}) ; \\
\square(\underline{X}, \bar{X}) & =(\underline{X}, \underline{X}) ; \\
\neg(\underline{X}, \bar{X}) & =(U \backslash X, \bar{U} \backslash \bar{X}) ; \\
0 & =(\emptyset, \emptyset) ; \\
1 & =(U, U) .
\end{aligned}
$$

Note that in [9], Jouni Järvinen and Sándor Radeleczki considered rough sets determined by quasiorders (reflexive and transitive binary relations), and showed that they form Nelson algebras.

Next we recall canonical n-valued Lukasiewicz-Moisil algebras (see [2], for example). They are structures

$$
\left(L_{n}, \vee, \wedge, \neg, \phi_{1}, \ldots, \phi_{n}, 0,1\right),
$$

where

$$
L_{n}=\left\{0, \frac{1}{n-1}, \frac{2}{n-1}, \ldots, \frac{n-2}{n-1}, 1\right\}
$$

and the operations are defined as

$$
\begin{aligned}
x \vee y & =\max \{x, y\}, \\
x \wedge y & =\min \{x, y\}, \\
\neg x & =1-x, \text { and } \\
\phi_{i}\left(\frac{j}{n-1}\right) & = \begin{cases}1 & \text { if } j \geq i \\
0 & \text { if } j<i .\end{cases}
\end{aligned}
$$

So-called Eukasiewicz t-norm is defined in $L_{n}$ by

$$
x \odot y=\max \{0, x+y-1\}
$$

and the corresponding to Eukasiewicz implication is defined as:

$$
x \rightarrow y= \begin{cases}1 & \text { if } x \leq y, \\ 1-x+y & \text { otherwise. }\end{cases}
$$


So, the structure

$$
\left(L_{n}, \vee, \wedge, \odot, \rightarrow, 0,1\right)
$$

is an involutive residuated lattice. Note that residuated lattices are considered in detail in Section 4.

Next we study in $L_{n}$ the properties of the operators $\diamond$ and $\square$ defined as in (1.2). This means that, for all $n \geq 3$,

$$
\diamond x=\min \{1,2 x\} \quad \text { and } \quad \square x=\max \{0,2 x-1\}
$$

in $L_{n}$. It is now easy to observe that

$$
\diamond x=1 \Longleftrightarrow x \geq \frac{1}{2} \quad \text { and } \quad \square x>0 \Longleftrightarrow x>\frac{1}{2} .
$$

In particular, if $n=3$, then $\phi_{1}=\diamond$ and $\phi_{2}=\square$. Hence, $\diamond x=0$ if $x=0$ and $\diamond x=1$ if $x=1$ or $x=\frac{1}{2}$. Similarly, $\square x=1$ if $x=1$ and $\square x=0$ if $x=0$ or $x=\frac{1}{2}$. For $n=4$, the values are presented in Table 3. So, in $L_{4}$,

$$
\phi_{1} \geq \diamond \geq \phi_{2} \geq \square \geq \phi_{3} \text {. }
$$

In addition, $\square 1=1$.

\begin{tabular}{c|ccccc} 
& $\phi_{1}$ & $\diamond$ & $\phi_{2}$ & $\square$ & $\phi_{3}$ \\
\hline 0 & 0 & 0 & 0 & 0 & 0 \\
$\frac{1}{3}$ & 1 & $\frac{2}{3}$ & 0 & 0 & 0 \\
$\frac{2}{3}$ & 1 & 1 & 1 & $\frac{1}{3}$ & 0 \\
1 & 1 & 1 & 1 & 1 & 1
\end{tabular}

Table 3. Values of $\phi_{1}, \diamond, \phi_{2}, \square, \phi_{3}$ for the case $n=4$.

It is also easy to observe that for $n \geq 5$,

$$
\phi_{1} \geq \diamond \geq \square \geq \phi_{n-1},
$$

but the other functions $\phi_{2}, \ldots, \phi_{n-2}$ are often incomparable with $\diamond$ and $\square$. For instance, if $n=6$, then

$$
\phi_{2}\left(\frac{1}{5}\right)=0<\frac{2}{5}=\diamond\left(\frac{1}{5}\right)
$$

but

$$
\phi_{2}\left(\frac{2}{5}\right)=1>\frac{4}{5}=\diamond\left(\frac{2}{5}\right) .
$$

The law of excluded middle $A \vee \neg A$ is valid in classical propositional logic, but not in most many-valued logics such as intuitionistic logic or $\mathrm{E}_{3}$. Note that in the literature expressions like $x \vee \neg x$ or $\neg(x \vee \neg x)$ are called abnormal objects and are intensively studied by several logicians. It is easy to see that, in $L_{n}$,

$$
x \vee \neg x=\left|x-\frac{1}{2}\right|+\frac{1}{2} .
$$

Thus,

$$
\diamond(x \vee \neg x)=1
$$


for any $x \in L_{n}$. On the other hand,

$$
\begin{aligned}
\square(x \vee \neg x) & =\max \left\{0,2\left(\left|x-\frac{1}{2}\right|+\frac{1}{2}\right)-1\right\} \\
& =\max \left\{0,2\left|x-\frac{1}{2}\right|\right\} \\
& =2\left|x-\frac{1}{2}\right| .
\end{aligned}
$$

This means that $\square(x \vee \neg x)=0$ if and only if $x=\frac{1}{2}$. This is possible only in $L_{n}$ with $n$ odd. In other words, if $n$ is even, then $\square(x \vee \neg x)>0$ for all $x \in L_{n}$.

In the next section, we will show that, in involutive residuated lattices,

$$
\square(x \rightarrow y) \rightarrow(\square x \rightarrow \square y)=1 \quad \text { and } \quad \square x \rightarrow x=1 .
$$

Thus, equations corresponding to axiom schemata $(\mathrm{K})$ and $(\mathrm{T})$ are valid in $L_{n}$.

In addition, by applying "truth tables", we can observe that the equation

$$
x \rightarrow \square \diamond x=1
$$

corresponding to schema (B) is valid in $L_{4}$. However, if $n \geq 5$, then for $x=\frac{1}{n-1}$,

$$
\diamond x=\frac{2}{n-1} \quad \text { and } \quad \square \diamond x=0 .
$$

Thus, the equation $x \rightarrow \square \diamond x=1$ corresponding to schema (B) is not valid in $L_{n}$ if $n \geq 5$.

Concerning (S4), for $n \geq 4$ and $x=\frac{n-2}{n-1}$,

$$
\square x=\frac{n-3}{n-1} \quad \text { and } \quad \square \square x=\max \left\{0, \frac{n-5}{n-1}\right\} .
$$

This means that $\square x \rightarrow \square \square x=1$ does not hold in $L_{n}$ if $n \geq 4$.

Finally, for $n \geq 4$, let $x=\frac{1}{n-1}$. Then

$$
\diamond x=\frac{2}{n-1} \quad \text { and } \quad \square \diamond x=\max \left\{0, \frac{5-n}{n-1}\right\} .
$$

So, the equation $\diamond x \rightarrow \square \diamond x=1$ corresponding to (S5) does not hold in $L_{n}$ for $n \geq 4$.

Our observations are summarized in the following proposition.

Proposition 3.4. The following propositions hold in canonical n-valued Eukasiewicz-Moisil algebras.

(a) For all $n \geq 3, \square 1=1$ in $L_{n}$.

(b) For all $n \geq 3$, the identities corresponding to axiom schemata $(\mathrm{K}),(\mathrm{T})$, and (D) hold in $L_{n}$.

(c) The identity corresponding to schema (B) holds only in $L_{3}$ and $L_{4}$.

(d) The identities corresponding to schemata (S4) and (S5) hold only in $L_{3}$.

As noted in [2], [3], it was observed by Alan Rose in 1965 that for $n \geq 5, n$ valued Łukasiewicz algebras do not correspond to $n$-valued Łukasiewicz logic. By the above observations, we can deduce the following proposition.

Proposition 3.5. Eukasiewicz 4-valued logic $\mathrm{E}_{4}$ is sound with respect to axioms $(\mathrm{K}),(\mathrm{T}),(\mathrm{B}),(\mathrm{D})$, that is, they are valid formulas. Additionally, the rules $(\mathrm{MP})$ and $(\mathrm{RN})$ preserve validity. 
As proved by Luisa Iturrioz in [8], one may always define in an $n$-valued Łukasiewicz-Moisil algebra $L$ a so-called Heyting implication by

$$
x \rightarrow y=y \vee \bigwedge\left\{\neg \phi_{i}(x) \vee \phi_{i}(y) \mid 1 \leq i \leq n-1\right\} .
$$

Therefore, $(L, \vee, \wedge, \rightarrow, 0,1)$ is a Heyting algebra (see also [5]). In a Heyting algebra, the pseudocomplement (negation) of $x$ is defined as $x \rightarrow 0$. It is obvious that for any $n$-valued Łukasiewicz-Moisil algebras, this induced pseudocomplement negation differs from the "original" negation $\neg$. Modalities (1.2) in Heyting algebras are studied in the following section.

\section{Hidden modalities in Residuated lattices and Heyting algebras}

In the previous sections, we defined $\diamond$ and $\square$ as truth functional modal operations in terms of negation and implication by setting

$$
\diamond A=\neg A \rightarrow A \quad \text { and } \quad \square A=\neg(A \rightarrow \neg A) .
$$

We also notice that, in the case of Boolean algebras, these operations are equal, that is, $\diamond x=\square x=x$.

In this section, our purpose is to study the properties of these hidden modalities in residuated lattices and Heyting algebras. First, we recall from [6] the definition of residuated lattices.

Definition 4.1. A residuated lattice is an algebra

$$
(L, \vee, \wedge, \odot, \rightarrow, 0,1)
$$

with four binary operations and two constants such that:

(i) The algebra $(L, \vee, \wedge, 0,1)$ is a lattice with the greatest element 1 and the least element 0 with respect to the lattice-ordering $\leq$ of $L$.

(ii) The algebra $(L, \odot, 1)$ is a commutative monoid, that is, $\odot$ is commutative and associative, and $1 \odot x=x$ for all $x$.

(iii) The operations $\odot$ and $\rightarrow$ form an adjoint pair, that is, for all $x, y, z$,

$$
z \leq(x \rightarrow y) \Longleftrightarrow x \odot z \leq y \text {. }
$$

Noti that we assume that, in residuated lattices, the operation $\odot$ is commutative, but in the literature, there exist definitions in which this assumption is omitted. Also in some definitions, the identity element 1 need not be the greatest element of the lattice $L$ with respect to its order $\leq$. In a residuated lattice,

$$
x \rightarrow(y \rightarrow z)=(x \odot y) \rightarrow z,
$$

for all elements $x, y, z$. We may define negation $\neg$ on $L$ by setting for all $x \in L$,

$$
\neg x=x \rightarrow 0 .
$$

It is easy to see that

(i) $\neg 0=(0 \rightarrow 0)=1$ and $\neg 1=1 \rightarrow 0=1 \odot(1 \rightarrow 0) \leq 0$, that is, $\neg 1=0$;

(ii) $x \odot x \leq x, x \odot \neg x=0$, and $x \leq y \Longleftrightarrow x \rightarrow y=1$;

(iii) $x \leq \neg \neg x$ and $\neg x=\neg \neg \neg x$ for any $x$.

In the sequel, we will need the following lemma. 
Lemma 4.2. Let $(L, \vee, \wedge, \odot, \rightarrow, 0,1)$ be a residuated lattice. Then, for all $x, y \in$ $L$,

$$
\neg \neg x \odot \neg \neg y \leq \neg \neg(x \odot y) .
$$

Proof. For all $x, y \in L$,

$$
\begin{aligned}
\neg \neg x \odot \neg \neg y \rightarrow \neg \neg(x \odot y) & =\neg \neg x \rightarrow(\neg \neg y \rightarrow \neg \neg(x \odot y)) \\
& =\neg \neg x \rightarrow(\neg(x \odot y) \rightarrow \neg y) \\
& =\neg(x \odot y) \rightarrow(\neg \neg x \rightarrow \neg y) \\
& =\neg(x \odot y) \rightarrow(y \rightarrow \neg x) \\
& =\neg(x \odot y) \odot y \rightarrow \neg x \\
& =\neg(x \odot y) \odot y \rightarrow(x \rightarrow 0) \\
& =\neg(x \odot y) \odot(y \odot x) \rightarrow 0 \\
& =0 \rightarrow 0 \\
& =1 .
\end{aligned}
$$

Hence, $\neg \neg x \odot \neg \neg y \leq \neg \neg(x \odot y)$.

Let us assume that, for a residuated lattice $(L, \vee, \odot, \rightarrow, \wedge, 0,1)$, the operators $\diamond$ and $\square$ are defined as in (1.2), that is,

$$
\diamond A:=\neg A \rightarrow A \quad \text { and } \quad \square A:=\neg(A \rightarrow \neg A) .
$$

Then, we can write the following proposition.

Proposition 4.3. Let $(L, \vee, \wedge, \odot, \rightarrow, 0,1)$ be a residuated lattice. Then, for all $x, y \in L$,

(i) $\square x=\neg \neg(x \odot x)$,

(ii) $\diamond 0=\square 0=0$ and $\diamond 1=\square 1=1$,

(iii) $x \rightarrow \diamond x=1, \neg \neg x \rightarrow \diamond x=1$, and $\square x \rightarrow \neg \neg x=1$,

(iv) $x \rightarrow y=1$ implies $\diamond x \rightarrow \diamond y=1$ and $\square x \rightarrow \square y=1$,

(v) $\square(x \rightarrow y) \rightarrow(\square x \rightarrow \square y)=1$.

Proof. (i) Obviously, $\neg(x \odot x)=(x \odot x) \rightarrow 0=x \rightarrow(x \rightarrow 0)=x \rightarrow \neg x$. This implies $\square x=\neg(x \rightarrow \neg x)=\neg \neg(x \odot x)$.

(ii) Now $\diamond 0=1 \rightarrow 0=0$ and $\square 0=\neg(0 \rightarrow 1)=\neg 1=0$. Similarly, $\diamond 1=0 \rightarrow$ $1=1$ and $\square 1=\neg(1 \rightarrow 0)=\neg 0=1$.

(iii) For all $x \in L, x \rightarrow \diamond x=x \rightarrow(\neg x \rightarrow x)=(x \odot \neg x) \rightarrow x=0 \rightarrow x=1$. Similarly, $\neg \neg x \rightarrow \diamond x=\neg \neg x \rightarrow(\neg x \rightarrow x)=(\neg \neg x \odot \neg x) \rightarrow x=0 \rightarrow x=1$. Because $x \odot x \leq x, \square x=\neg \neg(x \odot x) \leq \neg \neg x$. Thus, $\square x \rightarrow \neg \neg x=1$.

(iv) Let $x \rightarrow y=1$, that is, $x \leq y$. Thus, $\diamond x=\neg x \rightarrow x \leq \neg x \rightarrow y \leq \neg y \rightarrow$ $y=\diamond y$. Moreover, $x \leq y$ implies $x \odot x \leq x \odot y \leq y \odot y$. So, $\square x=\neg \neg(x \odot x) \leq$ $\neg \neg(y \odot y)=\square y$. 
(v) By Lemma 4.2,

$$
\begin{aligned}
\square(x \rightarrow y) \odot \square x & =\neg \neg((x \rightarrow y) \odot(x \rightarrow y)) \odot \neg \neg(x \odot x) \\
& \leq \neg \neg((x \rightarrow y) \odot(x \rightarrow y) \odot x \odot x) \\
& =\neg \neg(x \odot(x \rightarrow y) \odot x \odot(x \rightarrow y)) \\
& \leq \neg \neg(y \odot y) \\
& =\square y .
\end{aligned}
$$

Thus, $\square(x \rightarrow y) \leq \square x \rightarrow \square y$, that is, $\square(x \rightarrow y) \rightarrow(\square x \rightarrow \square y)=1$.

Let us now consider the above result from the viewpoint of modal logic. In [12], Michiro Kondo et al. introduced logic wUL, which is determined by the class of (commutative) residuated lattices. Statements (ii) and (v) of Proposition 4.3 are interesting since they mean that the hidden modalities $\square$ and $\diamond$ defined in wUL are normal in the sense that the necessitation rule (RN): "if $A$ is a theorem, then $\square A$ is a theorem" and the distribution axiom $(\mathrm{K}) \square(A \rightarrow B) \rightarrow(\square A \rightarrow \square B)$ can be included. By (iv),

(RM) $\frac{A \rightarrow B}{\square A \rightarrow \square B}$

is naturally a rule of inference. By Proposition 4.3(iii), $A \rightarrow \diamond A$, which is the dual of (T), may be adopted, but not (T) itself unless $\neg \neg A \rightarrow A$. In addition, we see that axiom $(\mathrm{D})$ is provable.

Let us now consider such residuated lattices that $x=\neg \neg x$ for all $x$; these are often called involutive residuated lattices.

Proposition 4.4. If $(L, \vee, \wedge, \odot, \rightarrow, 0,1)$ is an involutive residuated lattice, then, for all $x, y \in L$,

(i) $\square x=x \odot x$,

(ii) $\square x \rightarrow x=1$,

(iii) $\square \neg x=\neg \diamond x$ and $\diamond \neg x=\neg \square x$,

(iv) $\diamond(x \rightarrow y)=\square x \rightarrow \diamond y$.

Proof. Cases (i) and (ii) are obvious by Proposition 4.3.

(iii) $\square \neg x=\neg(\neg x \rightarrow \neg \neg x)=\neg(\neg x \rightarrow x)=\neg \nabla x$ and $\neg \square x=\neg \square \neg \neg x=$ $\neg(\neg \diamond \neg x)=\diamond \neg x$. Hence,

(iv) Because $x \rightarrow y=\neg(x \odot \neg y)$, we have $\neg(x \rightarrow y)=\neg \neg(x \odot \neg y)=x \odot \neg y$.

$$
\begin{aligned}
\diamond(x \rightarrow y) & =\neg \square \neg(x \rightarrow y) \\
& =\neg(\neg(x \rightarrow y) \odot \neg(x \rightarrow y)) \\
& =\neg((x \odot \neg y) \odot(x \odot \neg y)) \\
& =\neg((x \odot x) \odot(\neg y \odot \neg y)) \\
& =\neg(\square x \odot \square \neg y) \\
& =\neg(\square x \odot \neg \neg \square \neg y) \\
& =\neg(\square x \odot \neg(\diamond y)) \\
& =\square x \rightarrow \diamond y .
\end{aligned}
$$


Note that, if $(L, \vee, \wedge, \odot, \rightarrow, 0,1)$ is a residuated lattice such that $x=\neg \neg x$ for all $x \in L$, then

$$
\square x \rightarrow \square \square x=1 \Longleftrightarrow x \odot x=x \odot x \odot x \odot x .
$$

for all $x \in L$.

From the viewpoint of modal logic, Proposition 4.4(ii) means that axiom schema (T) may be adopted - $(\mathrm{T})$ is called the reflexivity axiom referring to reflexive accessibility relation in Kripke semantics of classical modal logic: axiom (T) holds if and only if, in the respective Kripke models, the accessibility relation is reflexive. As we showed in the previous section, the schemata (B), (S4), (S5) are not necessarily valid in all involutive residuated lattices.

Case (iii) of Proposition 4.4 means that $\square$ and $\diamond$ are dual, that is, each can be expressed by the other and negation. Hence, we have obtained so-called classical modal logic. Case (iv) means that $\diamond(A \rightarrow B)$ is logically equivalent to $\square A \rightarrow \diamond B$, which is interesting.

Equation (i) of Proposition 4.4 expresses the essential property of a so-called substantial modifier, because $\square x \leq x$ by (ii) - the connective $\square$ can be interpreted as a hedge "very" (cf. [7]).

Note that Proposition 4.4(iv) is an axiom introduced by Gisele Fischer Servi in [4] as an axiom of minimal intuitionistic modal logic IK establishing De Morgantype of duality between $\square$ and $\diamond$.

Example 4.5. Let us consider the connectives $\square$ and $\diamond$ in the setting of fuzzy sets. We take the real unit interval $[0,1]$ as the set of elements of the algebra and assume that the interval is ordered with its usual order $\leq$ of real numbers. Then, the ordered set $([0,1], \leq)$ is a lattice such that

$$
x \vee y=\max \{x, y\} \quad \text { and } \quad x \wedge y=\min \{x, y\} .
$$

Next we will extend this algebra to a residuated lattice. We consider the following three cases of defining $\odot$ that can be found, e.g, in [6].

(a) As in $L_{n}$, the Eukasiewicz t-norm is defined by

$$
x \odot y=\max \{0, x+y-1\} .
$$

The corresponding implication is defined so that $x \rightarrow y=1$ if $x \leq y$ and

$$
x \rightarrow y=1-x+y,
$$

otherwise. So, the negation is $\neg x=1-x$. In this setting, $\neg \neg x=x$ and the modal operators have the form

$$
\square x=\max \{0,2 x-1\} \quad \text { and } \quad \nabla x=\min \{1,2 x\} .
$$

(b) Gödel t-norm is defined by

$$
x \odot y=\min \{x, y\}=x \wedge y .
$$

The Gödel implication is defined by

$$
x \rightarrow y=y
$$


in the case $x>y$, otherwise the value is 1 . The corresponding negation is defined by $\neg 0=1$ and $\neg x=0$ for $x>0$. This implies that the modal operators are defined by

$$
\diamond 0=\square 0=0 \quad \text { and } \quad \nabla x=\square x=1 \text { for } x>0 .
$$

Note that, in this case, the algebra is in fact a Heyting algebra.

(c) Product t-norm is defined by

$$
x \odot y=x \cdot y .
$$

So-called Goguen implication is defined by $x \rightarrow y=y / x$ in case $x>y$, and the value is 1 otherwise. The complement is the same as in case (b).

In this setting, $\square x=\neg \neg x^{2}$. Unfortunately, since the complement is only two-valued, this leads to the same values of $\square$ and $\diamond$ as in case (b).

Let us mention that in [10] a plenty of t-norms of different kinds are presented, and it would be interesting to study modalities $\square$ and $\diamond$ defined by these norms, but this is beyond the scope of this paper.

A Heyting algebra $(L, \vee, \wedge, \rightarrow, 0,1)$ can be defined as a residuated lattice $(L, \vee, \wedge, \odot, \rightarrow, 0,1)$ such that $\odot$ coincides with $\wedge$. Heyting algebras are always distributive lattices. Note also that a Heyting algebra is a Boolean algebra if and only if $\neg \neg x=x$ for all $x$. For further properties of Heyting algebras, see [16], for example, where they are studied under the name pseudo-Boolean algebras.

Since $\odot$ and $\wedge$ are equal in Heyting algebras and the meet operation is idempotent, we have for all $x \in L$,

$$
\square x=\neg \neg x .
$$

We end this paper by the following proposition stating that, in Heyting algebras, the behaviour of $\square$ and $\diamond$ is similar to that in Boolean algebras: there is no difference between modalities $\diamond$ and $\square$, since they are both equal to the double negation.

Proposition 4.6. Let $(L, \vee, \wedge, \rightarrow, 0,1)$ be a Heyting algebra. Then, for all $x \in L$,

$$
\diamond x=\square x=\neg \neg x .
$$

Proof. It is enough to prove that (i) $\neg \neg x \rightarrow(\neg x \rightarrow x)=1$ and (ii) $(\neg x \rightarrow x) \rightarrow$ $\neg \neg x=1$.

(i) $\neg \neg x \rightarrow(\neg x \rightarrow x)=(\neg \neg x \wedge \neg x) \rightarrow x=0 \rightarrow x=1$.

(ii) $(\neg x \rightarrow x) \rightarrow \neg \neg x=(\neg x \rightarrow x) \rightarrow(\neg x \rightarrow 0)=((\neg x \rightarrow x) \wedge \neg x) \rightarrow 0=$ $(\neg x \wedge x) \rightarrow 0=1$.

By the previous proposition,

$$
x \leq \square \diamond x, \quad \square x=\square \square x, \quad \text { and } \quad \diamond x=\square \diamond x
$$

for all $x \in L$. This implies that the equations

$$
x \rightarrow \square \diamond x=1, \quad \square x \rightarrow \square \square x=1, \quad \text { and } \quad \diamond x \rightarrow \square \diamond x=1
$$

corresponding to schemata (B), (S4), and (S5) are valid.

It is well-known that Heyting algebras are models for intuitionistic logic, that is, a formula of intuitionistic logic is provable if and only if $A$ is valid in all Heyting algebras, meaning that $v(A)=1$ for all valuations on any Heyting algebra. This 
means that, if we define modalities $\square$ and $\diamond$ in intuitionistic logic as in (1.2), then all axioms (K), (T), (B), (D), (S4), (S5) considered above are provable and also rules $(\mathrm{RN})$ and $(\mathrm{RM})$ are admissible, because $\diamond, \square$, and the double negation are the same.

Note also that the well-known Glivenko Theorem states that if $\mathcal{F}$ is a set of propositional formulas and $A$ is a propositional formula, then $\mathcal{F}$ proves $A$ using classical logic if and only if $\mathcal{F}$ proves $\neg \neg A=\diamond A=\square A$ using intuitionistic logic.

\section{REFERENCES}

[1] R. Balbes and Ph. Dwinger, Distributive Lattices, University of Missouri Press, Columbia, Missouri, 1974.

[2] R. Cignoli, The algebras of Eukasiewicz many-valued logic: A historical overview, Lecture Notes in Comput. Sci. 4460 (2007), 69-83.

[3] R. Cignoli and M. S. de Gallego, The lattice structure of some Eukasiewicz algebras, Algebra Universalis 13 (1981), 315-328.

[4] G. Fischer Servi, Axiomatizations for some intuitionistic modal logics, Rend. Semin. Mat. Univ. Politec. Torino 42 (1984), 179-194.

[5] G. Georgescu, N-valued logics and Eukasiewicz-Moisil algebras, Axiomathes 16 (2006), $123-136$.

[6] P. Hájek, Metamathematics of Fuzzy Logic, Kluwer Academic Publishers, Dordrecht, 1998.

[7] Petr Hájek, On very true, Fuzzy Sets and Systems 124 (2001), 329-333.

[8] L. Iturrioz, Eukasiewicz and symmetrical Heyting algebras, MLQ Math. Log. Q. 23 (1977) 131-136.

[9] J. Järvinen and S. Radeleczki, Representation of Nelson algebras by rough sets determined by quasiorders, Algebra Universalis 66 (2011), 163-179.

[10] E. P. Klement, R. Mesiar and E. Pap, Triangular Norms, Kluwer Academic Publishers, Dordrecht, 2000.

[11] W. Kneale and M. Kneale, The Development of Logic, Oxford University Press, 1962.

[12] M. Kondo, M. F. Kawaguchi, M. Miyakoshi and O. Watari, Weak uninorm based logic and its filter theory, in: Proceedings of the 41st IEEE International Symposium on Multiple-Valued Logic (ISMVL 2011), 69-72.

[13] J. K. Mattila, Many-valuation, modality, and fuzziness, Stud. Fuzziness Soft Comput. 273 (2009), 231-260.

[14] P. Pagliani and M. Chakraborty, A Geometry of Approximation. Rough Set Theory: Logic, Algebra and Topology of Conceptual Patterns, Springer, 2008.

[15] Z. Pawlak, Rough sets, Internat. J. Comp. \& Inf. Sci. 11 (1982), 341-356.

[16] H. Rasiowa, An Algebraic Approach to Non-Classical Logics, North-Holland, Amsterdam, 1974.

[17] N. Rescher, Many-valued Logic, McGraw-Hill, New York, 1969. 
Jouni Järvinen, Sirkankuja 1, FI-20810 Turku, Finland e-mail: jouni.kalervo.jarvinen@gmail.com

Michiro Kondo, School of Information Environment, Tokyo Denki University, Inzai 270-1382, Japan

e-mail: kondo@sie.dendai.ac.jp

Jorma K. Mattila, Lappeenranta University of Technology, Department of Mathematics and Physics, P.O. Box 20, FI-53851 Lappeenranta, Finland

e-mail: jorma.mattila@lut.fi

Sándor Radeleczki, Institute of Mathematics, University of Miskolc, 3515 MiskolcEgyetemváros, Hungary

e-mail: matradi@uni-miskolc.hu 